\title{
High Resolution XPS study of oxide layers grown on Ge substrates
}

\author{
N. Tabet ${ }^{(a)}$, M. Faiz $^{(a)}$, N. M. Hamdan ${ }^{(b)}$ and Z. Hussain $^{(b)}$
}

(a) Surface Science Laboratory, Physics Department, KFUPM, Saudi Arabia.

(b) Lawrence Berkeley National Laboratory, Advanced Light Source, Berkeley, CA 94720

\begin{abstract}
High resolution X-ray Photoelectron Spectroscopy (XPS) was used to analyze thin layers of germanium oxide grown on germanium substrates under various conditions. The results reveal the presence of high density of electron states located at the oxide /germanium interface that lead to the energy band bending. The surface of native oxide layers and that of thin oxide layer grown under dry oxygen correspond to $\mathrm{GeO}_{2}$ composition. Under Ar etching, lower oxidation states were revealed. Short in-situ heat treatment at $\mathrm{T}=400{ }^{\circ} \mathrm{C}$ under ultra high vacuum leads to the removal of the oxide layer. In addition, the analysis of the layer grown at $\mathrm{T}=380^{\circ} \mathrm{C}$ under dry oxygen suggest that carbides form at the oxide/substrate interface.
\end{abstract}

PACS: 81.60. Cp, 79.60. Eq, 82.80. Pv

Keywords: germanium, surface oxidation, surface states, XPS, synchrotron.

\section{Introduction}

The semiconductor - oxide interface plays a crucial role in device fabrication and its properties affect in a large extent the devices performance. Most of the previous studies were dedicated to $\mathrm{Si} / \mathrm{SiO} 2$ because of its technological importance. Despite the high carrier mobility in germanium and the possibility to obtain nearly perfect crystals, this semiconductor has been discarded for many applications mainly because of its cost and the instability of its oxide. However, germanium remains an attractive candidate for manufacturing complementary metal oxide semiconductor (CMOS) elements and metal insulator semiconductor (MIS) devices in very large scale integrated (VLSI) circuits [1]. Few papers have been recently published on the use of XPS to analyze oxidized germanium surfaces and the bonding chemistry of germanium oxides [2-7]. In a previous work, we have used conventional XPS to investigate the growth kinetics of thin oxide layers on germanium substrates under various experimental conditions [8]. In this work, we have used high resolution XPS using synchrotron radiation to analyze both the native oxide grown on chemically etched germanium surface and oxide layers obtained by dry oxidation of germanium substrate under pure oxygen atmosphere.

\section{Experimental}

Germanium samples were mechanically polished using diamond paste down to $1 \mu \mathrm{m}$ size. Then they were cleaned using acetone then immersed in a CP4 solution ( HF: $\mathrm{HNO} 3$ : $\mathrm{CH} 3 \mathrm{COOH}, 15: 10: 14$ by volume) for 3 minutes and rinsed with distilled water. Two samples were stored under air for one year. A third sample was oxidized at 
$\mathrm{T}=380^{\circ} \mathrm{C}$ for 25 minutes, under $400 \mathrm{mTorr}$ of dry oxygen. The XPS measurements were carried out at 9.3.2 beam line of the Advanced Light Source (ALS) of the Lawrence Berkeley National Laboratory (LBNL, USA). The incident photon energy was varied between 300 and $650 \mathrm{eV}$. A state-of-the-art electron energy analyzer (type Scienta) was used. Taking into account the resolving power of the monochromator and our experimental conditions, the overall energy resolution was better than $0.2 \mathrm{eV} . \mathrm{C} 1 \mathrm{~s}, \mathrm{Ge} 3 \mathrm{~d}$ and O1s lines were recorded from the as grown oxide layers and after argon etching cycles of various durations. The $\mathrm{C} 1 \mathrm{~s}$ line of $284.5 \mathrm{eV}$ binding energy was used as a reference to correct the binding energies for the charge shift. In situ heat treatments were carried out under ultra high vacuum by electron bombardment of the back surface of the samples in the XPS analysis chamber.

\section{Analysis of the native oxide}

Figure 1 shows the Ge3d line recorded from a layer of native oxide grown on a chemically etched germanium surface during storage under air for one year. The incident photon beam was focused down to $1 \mathrm{~mm}$ diameter. The photon energy was $600 \mathrm{eV}$. The broad peak located at $32.4 \mathrm{eV}$ stems from the oxide layer. The second small peak located at $28.9 \mathrm{eV}$ originates from the non oxidized atoms of the substrate. The spectrum 2 in the figure was obtained after heat treatment at $\mathrm{T}=400^{\circ} \mathrm{C}$ under ultra high vacuum $\left(10^{-9}\right.$ mbar) for $15 \mathrm{~min}$. One can clearly observe that the signal stemming from the oxide layer has vanished while the peak originating from the substrate increases drastically indicating that the native oxide has been removed as a result of the heat treatment. One can also observe a $0.6 \mathrm{eV}$ shift of the substrate signal towards higher binding energy (from $28.9 \mathrm{eV}$ to $29.5 \mathrm{eV}$ ). This shift results from the band bending that occurs at the interface oxide/substrate. In N-type samples, the band binding leads to the reduction of the distance between the Fermi level, which is flat, and the core level at the surface. It should be pointed out that a $0.5 \mathrm{eV}$ shift of the Ge3d line was observed in ref. 2 but in the opposite direction, i.e. towards higher binding energy. As p-type samples were used in ref. 2 , it is expected that the band bending occurs in the opposite direction to that observed in n-type samples. Therefore, our observations are qualitatively consistent with those reported in ref.2.

Subsequently argon etching (curve 3 ) had no significant effect except a less visible shoulder corresponding to the $\mathrm{Ge}_{3 / 2} \mathrm{~d}_{3 / 2}$ line due to the structural damage resulting from the ion bombardment. Notice that the shoulder is back in the picture after a heat treatment at $\mathrm{T}=400^{\circ} \mathrm{C}$ (curve 4). Curve 5 was obtained with a better energy resolution using $300 \mathrm{eV}$ photon energy and shows clearly the two components of the Ge3d line.

A second sample with a native oxide layer grown in similar conditions was analyzed after successive Ar etching cycles. Figure 2 shows the evolution of the Ge3d line. In addition to the shift of the signal originating from the substrate (from 28.8 to $29.2 \mathrm{eV}$ ) similar to that observed after the removal of the oxide layer in the previous sample, one can observe a $1 \mathrm{eV}$ shift of the signal stemming from the oxide layer towards lower binding energies (from 32. 4 to $31.4 \mathrm{eV}$, Fig. 2, curve 4). A simple explanation for this observation is to assume a reduction of the oxide under the ion bombardment. In order to check this interpretation, we have analyzed a $\mathrm{GeO}_{2}$ amorphous sample after successive Ar etching cycle using a conventional XPS instrument (ESCALAB MKII). The sample was prepared by a vapor condensation technique using a solar furnace [9]. Surprisingly, 
the results showed a very stable oxide since no shift of the Ge3d line was observed after $20 \mathrm{~min}$. argon sputtering using $3 \mathrm{keV}$ ion energy. Therefore, we conclude that the inner layers of the native oxide may include lower oxidation states of germanium such as $\mathrm{Ge}^{3+}$ and $\mathrm{Ge}^{2+}$. However, considering the small thickness of the oxide layers, one cannot completely exclude a possible effect of the substrate that would contribute to the formation of lower oxidation states during the ion bombardment.

In the following section we present similar results obtained by analyzing an oxide layer formed by dry oxidation of a germanium sample.

Figure 3 shows two XPS spectra of the valence band region. The spectrum 1 was obtained from the surface covered by the native oxide. The second curve was recorded after Ar etching of 160 minute duration using $1.5 \mathrm{keV}$ ion energy followed by a heat treatment under ultra high vacuum at $\mathrm{T}=315^{\circ} \mathrm{C}$ for $20 \mathrm{~min}$. The three germanium bands, commonly called I, II and III [10] are clearly visible, preceded by a strong broad peak. The spectrum 2 obtained after removal of the oxide layer, shows that the three germanium bands remained while the broad strong peak vanished. The latter was assigned to electron states located at the oxide/substrate interface. These are the charged states that are responsible of the band bending. The high intensity of the peak indicates a high density of the electron states. The absence of the signal stemming from these states after the removal of the oxide layer is consistent with the shift of the Ge3d core level stemming from the substrate that was discussed above.

Analysis of an oxide layer obtained by dry oxidation at $\mathrm{T}=380^{\circ} \mathrm{C}$, under dry oxygen: Figure 4 shows the evolution of Ge3d line after successive Ar etching cycles. The first four cycles were carried out using $1 \mathrm{keV}$ ion energy and a gas pressure $\mathrm{P}_{\mathrm{Ar}}=10^{-7} \mathrm{mbar}$. After 2 hour etching the conditions were changed into $1.5 \mathrm{keV}$ ion energy and $\mathrm{P}_{\mathrm{Ar}}=$ $5 \times 10^{-6}$ mbar. One can notice similar results to that observed in the case of the native oxide, in particular, the $1 \mathrm{eV}$ shift of the signal stemming from the oxide layer $\left(\mathrm{Ge} 3 \mathrm{~d}^{\mathrm{Ox}}\right)$. However, we observe a more progressive shift of the signal originating from the substrate. This could be a result of the non uniformity of the oxide layer that leads to a progressive removal of islands of oxide during sputtering.

We have also systematically recorded the evolution of the $\mathrm{C} 1 \mathrm{~s}$ spectrum as it was used to correct the binding energies for the charge shift (Fig.5). The counts rate was very low as the ion etching progressed. Unfortunately we did not increase the counting time to improve the statistics. However, the spectra show a clear shoulder located at $283 \mathrm{eV}$ binding energy as the interface oxide/substrate is reached. This shoulder could be assigned to carbides particles present at the oxide/substrate interface. This is a surprising result because it contradicts a common belief that carbon does not form carbides by reacting with germanium $[2,11]$ Further investigations are needed in order to confirm a possible formation of carbides as a result of our conditions of surface treatment and oxidation.

\section{Conclusion}

High Resolution XPS was used to analyze thin oxide layers grown on germanium substrates. The results revealed the presence of a high density of electron states located at the oxide/germanium interface that leads to about $0.6 \mathrm{eV}$ band bending. The composition 
of the surface of both native oxides and oxide layer obtained by dry oxidation of a germanium substrate corresponds to that of $\mathrm{GeO}_{2}$ (i.e. only $\mathrm{Ge}^{4+}$ signal was present). A $1 \mathrm{eV}$ shift of the signal originating from the oxidized germanium atoms was observed towards the lower binding energy after successive Ar etching cycles indicating the presence of lower oxidation states. In addition, a signal assigned to carbides was detected at the oxide/substrate interface in the sample that was oxidized under dry oxygen atmosphere.

\section{Acknowledgments}

This work was partially supported by the U.S. Department of Energy under Contract No. DE-AC03-76SF00098. Drs Faiz and Tabet would like to thank King Fahd University of Petroleum and Minerals for its support.

\section{References}

1. L. Chun-rong and L. Ling, J. Phys. D: Appl. Phys. 22 (1989), 1169.

2. J.S. Hovis, R. J. Hamers, and C. M. Greenlief Surf. Sc. 440 (1999) L815.

3. T. Geegan and G. Hughes, Appl. Surf. Sci. , 123/124 (1998) 66.

4. T. Barr, M. Mohsenian and L.M. Chen, Appl. Surf. Sci. 51 (1991)71.

5. N. Tabet, J. Al-Sadah and M. Salim, Surf. Sci. Rev. and Letters, 6, 6 (1999) 1053.

6. D. Schmeisser, R.D. Schnell, A. Bogen, F.J. Himpsel, D. Rieger, G. Landgren and J.F. Morar, Surf. Sci. 172 (1986) 455.

7. Y. Wei, J. Sullivan and S.O Said, Vacuum, 45, 5 (1994) 597.

8. N. Tabet, J. Al-Sadah and M. Salim, Surf. Sci. Rev. and Letters, 6, 6 (1999) 1053.

9. The sample was prepared with the collaboration of Dr. C.Monty, IMP-CNRS, Odeillo, France.

10. Y. Yu and M. Cardona, in 'Fundamentals of Semiconductors, Physics and Materials Properties", Springer-Verlag, Berlin, heidelberg, New York, 1996, page 429.

11. V.I. Davydov, Germanium, Gordon and Breach, New York, 1966. 


\section{Figures captions}

Fig. 1 Oxide layer grown at room temperature on a CP4 etched surface. Evolution of Ge3d line Incident Photon energy: $600 \mathrm{eV}$, as grown layer (1), after heat treatment under vacuum $10^{-9}$ mbar, $\mathrm{T}=400{ }^{\circ} \mathrm{C}, 15 \mathrm{~min}$. (2); then Ar etching, $1.5 \mathrm{keV}, \mathrm{P}_{\mathrm{Ar}}=2.0^{-5}$ mbar, $20 \mathrm{~min}$. (3); then heat treatment under vacuum $\mathrm{T}=400{ }^{\circ} \mathrm{C}, 20 \mathrm{~min}$. (4). The spectrum (5) corresponds to the same conditions as (4) but using $300 \mathrm{eV}$ incident energy. Notice that $\mathrm{Ge} 3 \mathrm{~d}_{5 / 2}$ and $\mathrm{Ge} 3 \mathrm{~d}_{3 / 2}$ lines presenting a $0.53 \mathrm{eV}$ splitting are well resolved.

Fig. 2. Native oxide layer grown at room temperature on a $\mathrm{CP} 4$ etched surface. Evolution of Ge3d line after successive Ar etching cycles. Incident Photon energy: $600 \mathrm{eV}$. As grown layer (1); after Ar etching, $1 \mathrm{keV}, \mathrm{P}_{\mathrm{Ar}}=10^{-5} \mathrm{mbar}, 50 \mathrm{~min}$. (2); then same conditions, $100 \mathrm{~min}(3)$; then $1.5 \mathrm{keV}, \mathrm{P}_{\mathrm{Ar}}=10^{-5} \mathrm{mbar}, 1 \mathrm{~h}$.

\section{Fig.3}

Valence band of germanium surface covered by the native oxide (1) and after Ar etching, $1.5 \mathrm{keV}, 160 \mathrm{~min}$.; then annealing under ultra high vacuum, $\mathrm{T}=315^{\circ} \mathrm{C}, 20 \mathrm{~min}$. (2).

Fig.4 . Evolution of the Ge3d signal after successive Ar etching cycles of an oxidized Ge surface. Oxide layer grown by thermal oxidation of $(011) \mathrm{Ge}$ substrate, $\mathrm{T}=380{ }^{\circ} \mathrm{C}, \mathrm{t}=$ 25 min. , $\mathrm{P}=400 \mathrm{~m}$ Torrs, dry oxygen. Incident photon energy: $650 \mathrm{eV}$. As grown layer (1); after ion etching, $1 \mathrm{keV}, \mathrm{P}_{\mathrm{Ar}}=10^{-7} \mathrm{mbar}$, etching time $33 \mathrm{~min}$. (2); $58 \mathrm{~min}$. (3), 103 min. (4). After 2 hours of Ar etching the conditions were changed into $1.5 \mathrm{keV}$ ion energy, $\mathrm{P}_{\mathrm{Ar}}=5.10^{-6}$ mbar. $10 \mathrm{~min}$. (5); $15 \mathrm{~min}$. (6); $20 \mathrm{~min} .(7) ; 45 \mathrm{~min}$. (8); $62 \mathrm{~min}$. (9). Notice that the last spectrum (9) was obtained using $300 \mathrm{eV}$ photon energy. The Ge $3 \mathrm{~d}_{5 / 2}$ and $\mathrm{Ge} 3 \mathrm{~d}_{3 / 2}(0.53 \mathrm{eV}$ splitting) are well resolved.

Fig.5. Evolution of the carbon signal after successive Ar etching cycles of an oxidized Ge surface. Oxide layer grown by thermal oxidation of $(011) \mathrm{Ge}$ substrate, $\mathrm{T}=380{ }^{\circ} \mathrm{C}, \mathrm{t}=$ 25 min., $\mathrm{P}=400 \mathrm{mTorrs}$, dry oxygen. Incident photon energy: $650 \mathrm{eV}$. As grown layer (1); after Ar etching with $1 \mathrm{keV}$ ion energy, $\mathrm{P}_{\mathrm{Ar}}=10^{-7}$ mbar, 33 min. (2); 58 min. (3). After 2 hours of Ar etching the conditions were changed into $1.5 \mathrm{keV}$ ion energy, $\mathrm{P}_{\mathrm{Ar}}=$ 5. $10^{-6}$ mbar. Etchnig time $10 \mathrm{~min}$. (4); $20 \mathrm{~min}$. (5); $45 \mathrm{~min} .(6) ; 62 \mathrm{~min}$. (7). Notice the small signal assigned to carbides that appears as a shoulder indicated by the arrow localized at $282.8 \mathrm{eV}$ binding energy. 


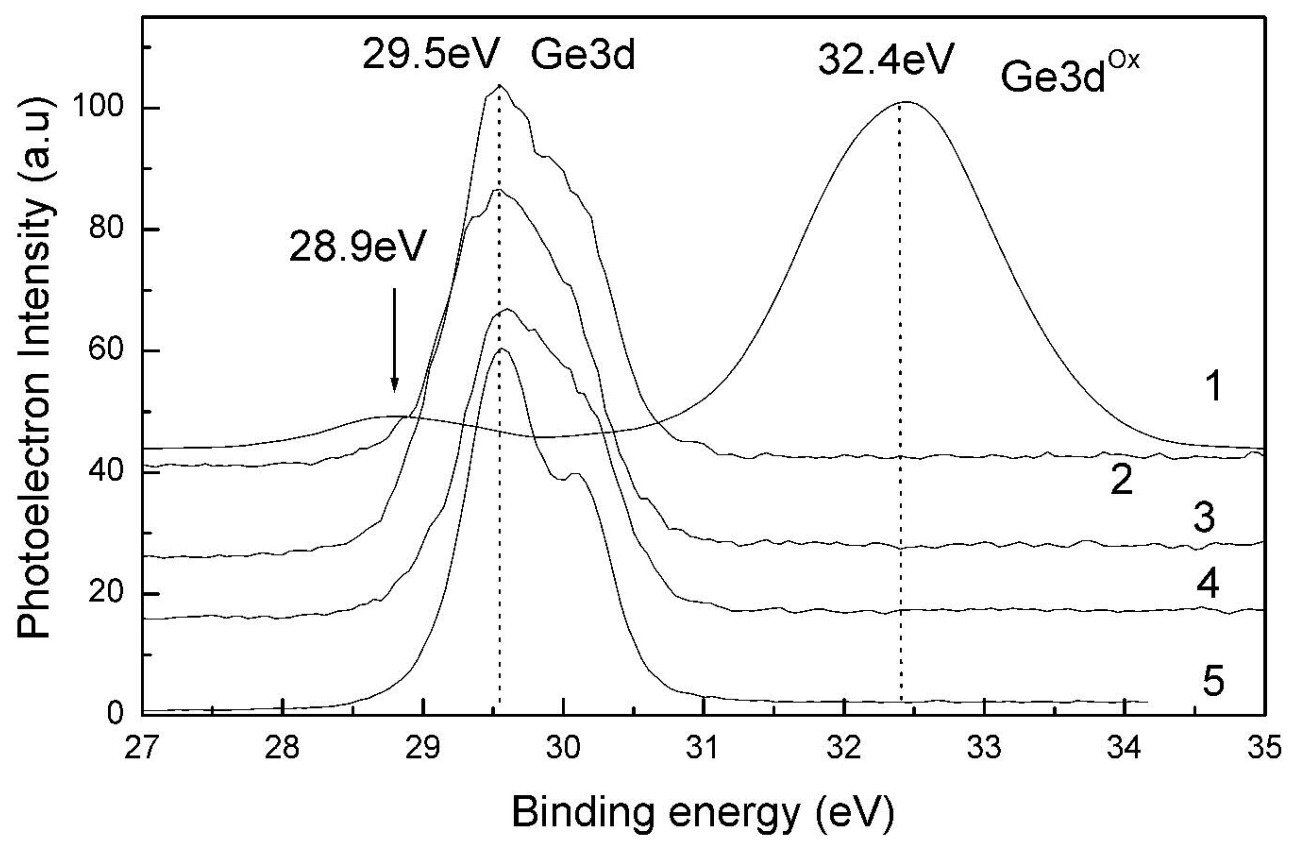

Fig. 1 


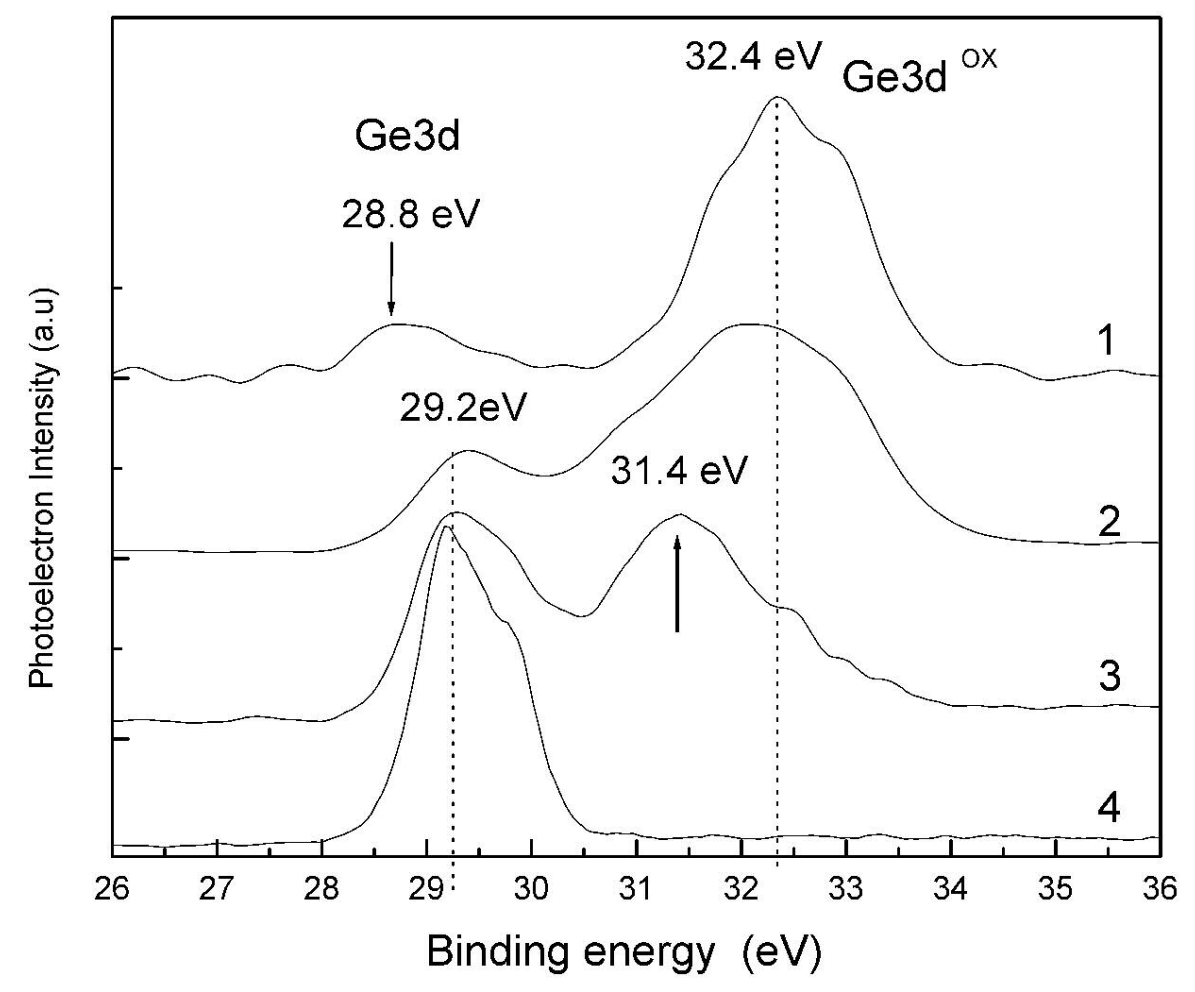

Fig. 2 


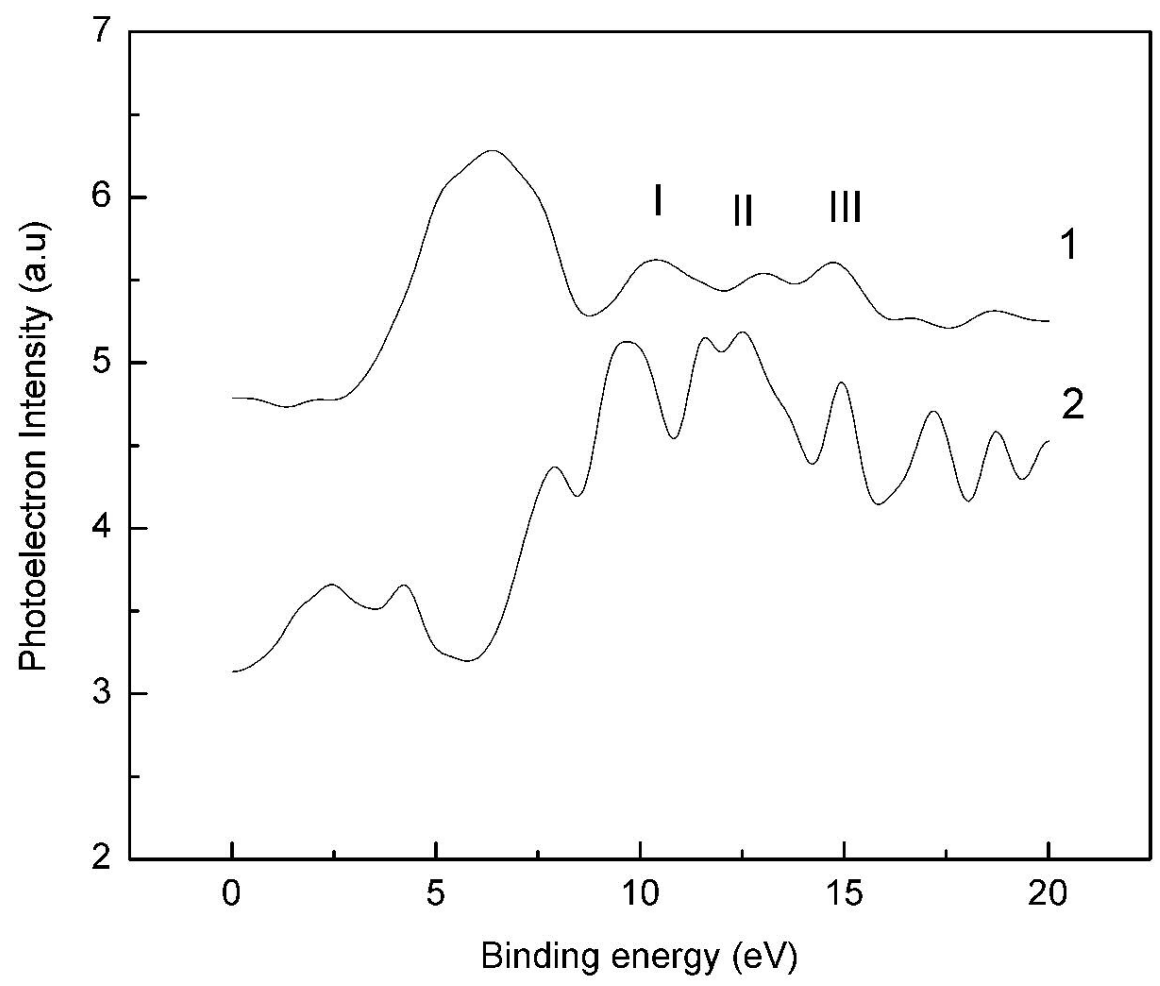

Fig. 3 


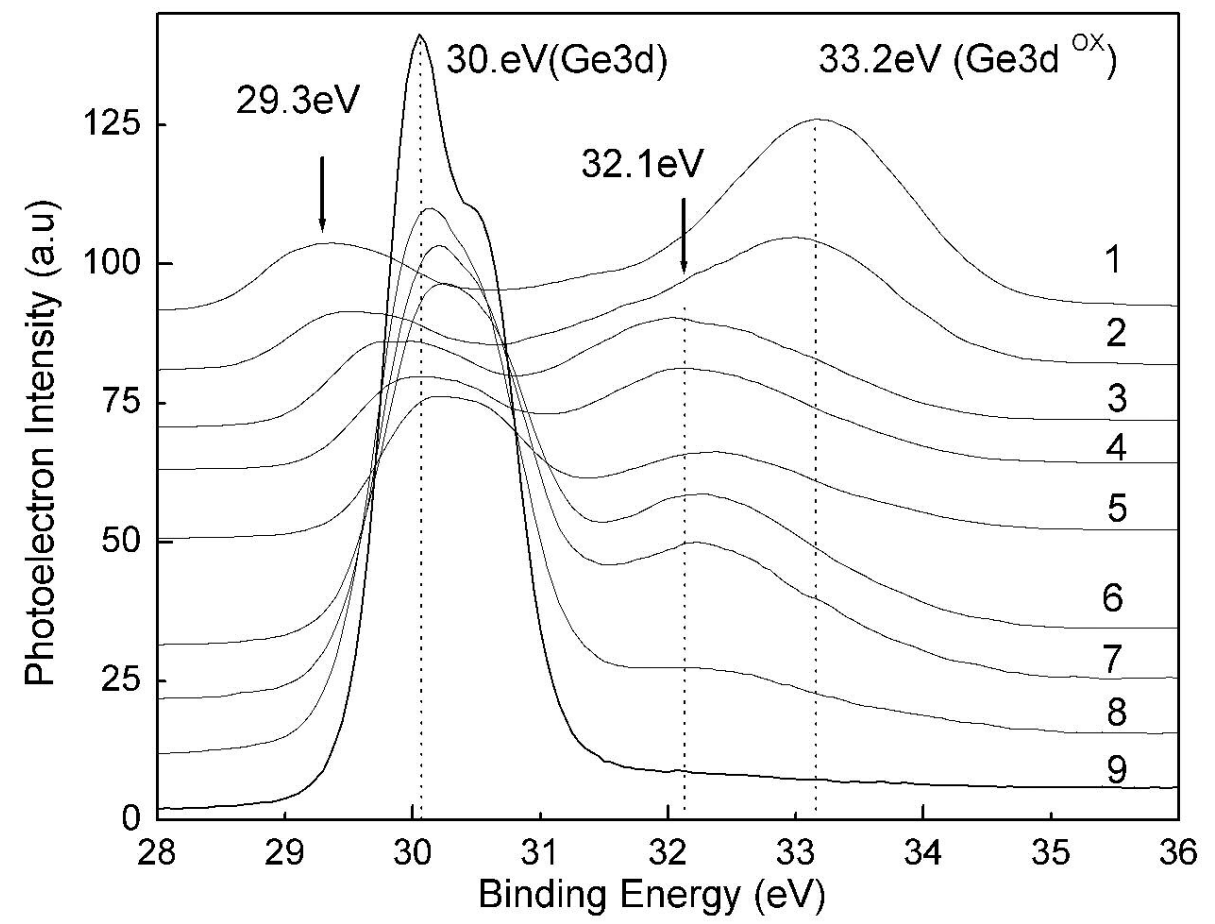

Fig. 4 


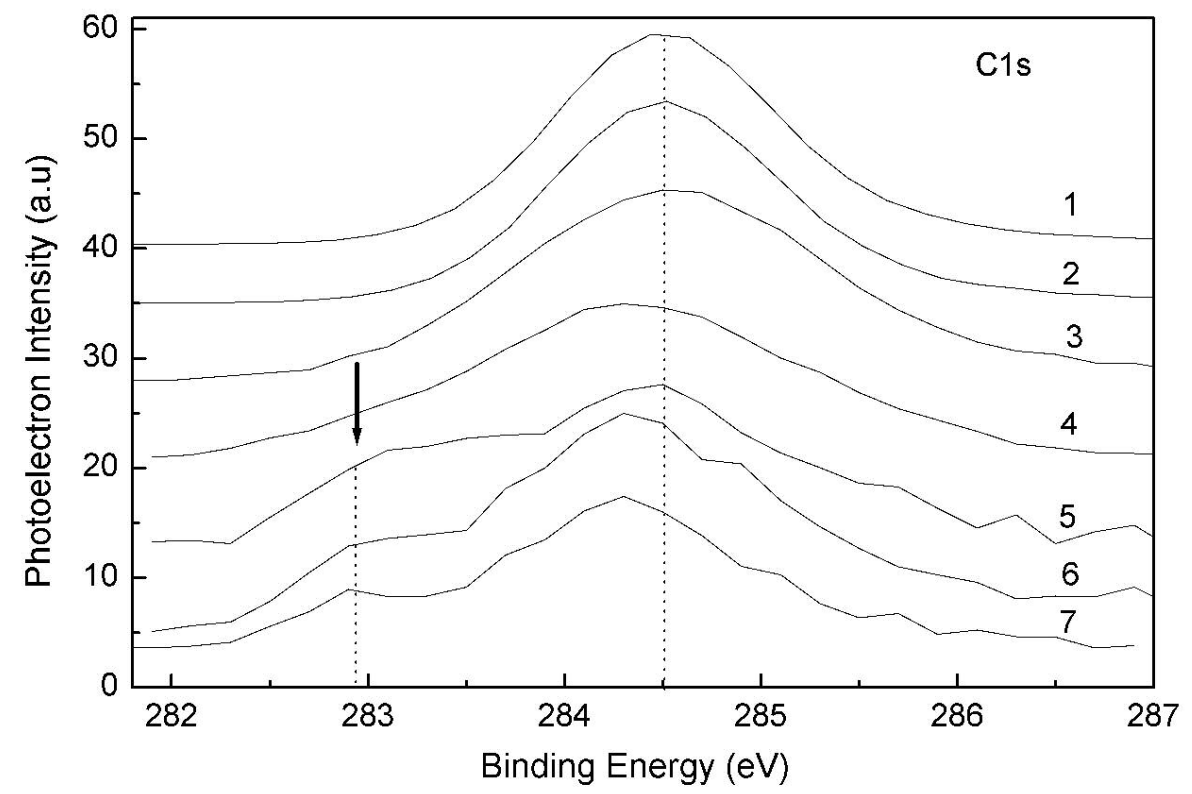

Fig. 5 